\title{
Spastic paraplegia type 7 associated with a broad clinical phenotype
}

\author{
Luke Francis $\mathrm{O}^{\prime}$ Donnell ${ }^{1} \cdot$ Michael Hennessy ${ }^{1}$
}

Received: 25 February 2020 / Accepted: 28 February 2020 / Published online: 10 March 2020

(C) Royal Academy of Medicine in Ireland 2020

Dear Editor,

The p.Ala510Val mutation in the spastic paraplegia type 7 (SPG7) gene has been identified as a common mutation causing hereditary spastic paraparesis (HSP) $[1,2]$. SPG7 is reported as an autosomal recessive disease [3]. The phenotype can be broad encompassing upper limb hyperreflexia and ophthalmoplegia [3].

An example is a case of a 65 -year-old male who presented with an unusual phenotype of cerebellar ataxia and bilateral ptosis and was found to be compound heterozygous for the p.Ala510Val and p.Pro749Leu mutation.

The patient in question first noticed symptoms at 30 years of age. He had difficulties with coordination and dysarthria. He also developed bilateral sensorineural hearing loss. Symptoms progressed slowly over the years. His brother developed similar symptoms that have also progressed. He reports that his father had problems with coordination.

On examination, his speech was dysarthric. His gait demonstrated ataxia. He was noted to have bilateral non-fatigable ptosis. Ocular examination demonstrated horizontal and vertical nystagmus with a complex ophthalmoplegia. There was lower limb dysmetria with hyperreflexia and crossed reflexes.

Brain MRI demonstrated extensive cerebellar atrophy of the vermis. Muscle biopsy was negative for mitochondrial disease changes. Genetic testing revealed that the patient was compound heterozygous for the c.1529C $>\mathrm{T}$, p.Ala510Val mutation in exon 11 and the c.2246C $>\mathrm{T}$ p.Pro749Leu mutation in exon 17 of the SPG7 gene.

SPG7 is linked to the maintenance of mitochondrial DNA [3]. SPG7 is caused by mutations in the gene that code for the protein paraplegin [3]. Paraplegin is involved in the processing of other mitochondrial proteins where it is speculated that if mutated, it may lead to mitochondrial DNA damage by impairing the function of other mitochondrial proteins [3].

While our patient did not have any mitochondrial DNA abnormalities in his muscle biopsy, this does not rule out a mitochondrial pathology as muscle biopsies can be negative due to uneven distribution of mitochondrial DNA defects in muscle fibres [3].

This highlights the diverse phenotype associated with SPG7. We speculate that there is an associated defect in mitochondrial DNA homeostasis that accounts for this [3]. We suggest that in cases of bilateral ptosis and ophthalmoplegia where ocular myasthenia gravis has been outruled, SPG7 should be considered in the differential diagnosis.

\section{References}

1. Roxburgh RH, Marquis-Nicholson R, Ashton F et al (2013) The p.Ala510Val mutation in the SPG7 (paraplegin) gene is the most common mutation causing adult onset neurogenetic disease in patients of British ancestry. J Neurol 260(5):1286-1294

2. Mihaylova P, Murphy S, Walsh R (2017) SPG7-related ataxia in the Irish National Ataxia clinic cohort. J Neurol Neurosurg Psychiatry 88(suppl 1):A1-A83

3. Wedding IM, Koht J, Tran GT et al (2014) Spastic Paraplegia Type 7 Is Associated with Multiple Mitochondrial DNA Deletions. PLoS ONE 9(1):e86340

Publisher's note Springer Nature remains neutral with regard to jurisdictional claims in published maps and institutional affiliations.

Luke Francis O’Donnell

odonnell4ie@hotmail.com

1 Department of Neurology, University Hospital Galway, Galway, Ireland 